\title{
Geometrical Validity of Curvilinear Finite Elements
}

\author{
A. Johnen ${ }^{1}$, J.-F. Remacle ${ }^{2}$ and C. Geuzaine ${ }^{1}$ \\ 1 Université de Liège, Department of Electrical Engineering and Computer Science, \\ Montefiore Institute B28, Grande Traverse 10, 4000 Liège, Belgium \\ ${ }^{2}$ Université catholique de Louvain, Institute of Mechanics, Materials and Civil \\ Engineering (iMMC), Place du Levant 1, 1348 Louvain-la-Neuve, Belgium
}

\begin{abstract}
In this paper, we describe a way to compute accurate bounds on Jacobian determinants of curvilinear polynomial finite elements. Our condition enables to guarantee that an element is geometrically valid, i.e., that its Jacobian determinant is strictly positive everywhere in its reference domain. It also provides an efficient way to measure the distortion of curvilinear elements. The key feature of the method is to expand the Jacobian determinant using a polynomial basis, built using Bézier functions, that has both properties of boundedness and positivity. Numerical results show the sharpness of our estimates.
\end{abstract}

Keywords: Finite element method; high-order methods; mesh generation; Bézier functions

\section{Introduction}

There is a growing consensus in the Finite Element community that higher-order discretization methods will replace at some point the solvers of today, at least for part of their applications. These high-order methods require a good accuracy of the geometrical discretization to be accuratein other words, such methods will critically depend on the availability of high-quality curvilinear meshes.

The usual way of building such curvilinear meshes is to first generate a straight sided mesh. Then, mesh entities that are classified on the curved boundaries of the domain are curved accordingly [1-3]. Some internal mesh

Email address:

a.johnen@ulg.ac.be, jean-francois.remacle@uclouvain.be, cgeuzaine@ulg.ac.be (A. Johnen ${ }^{1}$, J.-F. Remacle ${ }^{2}$ and C. Geuzaine ${ }^{1}$ ) 
entities may be curved as well. If we assume that the straight sided mesh is composed of well shaped elements, curving elements introduces a "shape distortion" that should be controlled so that the final curvilinear mesh is also composed of well shaped elements. The optimization of the shape distortion is a computationally expensive operation, especially when applied globally over the full mesh. It is thus crucial to be able to get fast and accurate bounds on the distortion in order to 1) evaluate the quality of the elements during the optimization process; and 2) reduce the sets of elements to be optimized, so that the optimization can be applied locally, i.e., only where it is necessary.

In this paper we present a method to analyze curvilinear meshes in terms of their elementary Jacobian determinants. The method does not deal with the actual generation/optimization of the high order mesh. Instead, it provides an efficient way to guarantee that each curvilinear element is geometrically valid, i.e., that its Jacobian determinant is strictly positive everywhere in its reference domain. It also provides a way to measure the distortion of the curvilinear element. The key feature of the method is to adaptively expand the elementary Jacobian determinants in a polynomial basis that has both properties of boundedness and positivity. Bézier functions are used to generate these bases in a recursive manner. The proposed method can be either used to check the validity and the distortion of an existing curvilinear mesh, or embedded in the curvilinear mesh generation procedure to assess the validity and the quality of the elements on the fly. The algorithm described in this paper has been implemented in the open source mesh generator Gmsh [4], where it is used in both ways.

\section{Curvilinear Meshes, Distortion and Bounds on Jacobian De- terminants}

Let us consider a mesh that consists of a set of straight-sided elements of order $p$. Each element is defined geometrically through its nodes $\mathbf{x}_{i}$, $i=1, \ldots, N_{p}$ and a set of Lagrange shape functions $\mathcal{L}_{i}^{(p)}(\boldsymbol{\xi}), i=1, \ldots, N_{p}$. The Lagrange shape functions (of order $p$ ) are based on the nodes $\mathbf{x}_{i}$ and allow to map a reference unit element onto the real one:

$$
\mathbf{x}(\boldsymbol{\xi})=\sum_{i=1}^{N_{p}} \mathcal{L}_{i}^{(p)}(\boldsymbol{\xi}) \mathbf{x}_{i}
$$

The mapping $\mathbf{x}(\boldsymbol{\xi})$ should be bijective, which means that it should admit an inverse. This implies that the Jacobian determinant $\operatorname{det} \mathbf{x}_{, \boldsymbol{\xi}}$ has to be strictly 


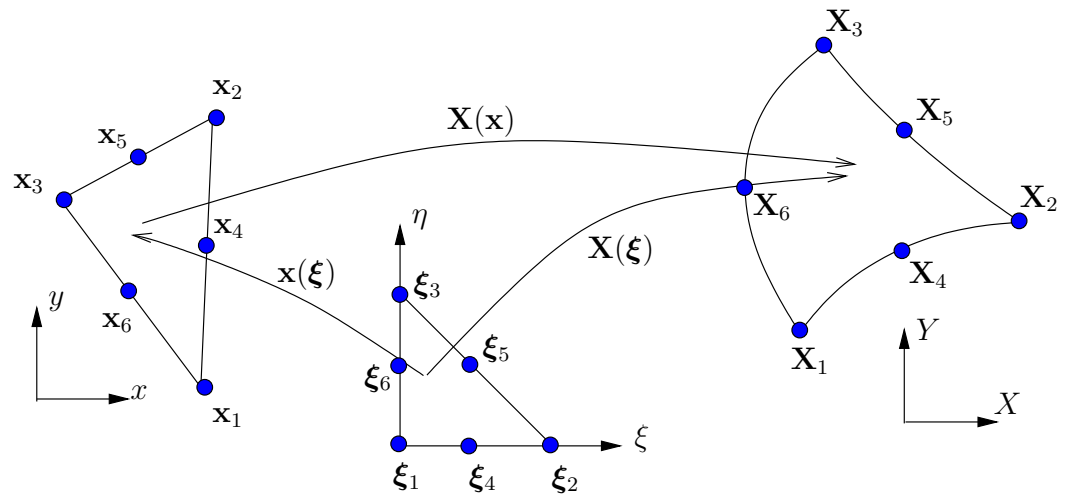

Figure 1: Reference unit triangle in local coordinates $\boldsymbol{\xi}=(\xi, \eta)$ and the mappings $\mathbf{x}(\boldsymbol{\xi})$, $\mathbf{X}(\boldsymbol{\xi})$ and $\mathbf{X}(\mathbf{x})$.

positive. In all what follows we will always assume that the straight-sided mesh is composed of well-shaped elements, so that the positivity of $\operatorname{det} \mathbf{x}_{, \boldsymbol{\xi}}$ is guaranteed. This standard setting is presented on Figure 1 (left) for the quadratic triangle.

Let us now consider a curved element obtained after application of the curvilinear meshing procedure, i.e., after moving some or all of the nodes of the straight-sided element. The nodes of the deformed element are called $\mathbf{X}_{i}, i=1 \ldots N_{p}$, and we have

$$
\mathbf{X}(\boldsymbol{\xi})=\sum_{i=1}^{N_{p}} \mathcal{L}_{i}^{(p)}(\boldsymbol{\xi}) \mathbf{X}_{i}
$$

Again, the deformed element is assumed to be valid if and only if the Jacobian determinant $J(\boldsymbol{\xi}):=\operatorname{det} \mathbf{X}_{, \boldsymbol{\xi}}$ is strictly positive everywhere over the $\boldsymbol{\xi}$ reference domain. The Jacobian determinant $J$, however, is not constant over the reference domain, and computing $J_{\min }:=\min _{\boldsymbol{\xi}} J(\boldsymbol{\xi})$ is necessary to ensure positivity.

The approach that is commonly used is to sample the Jacobian determinant on a very large number of points. Such a technique is however both expensive and not fully robust since we only get a necessary condition. In this paper we follow a different approach: because the Jacobian determinant $J$ is a polynomial in $\boldsymbol{\xi}, J$ can be interpolated exactly as a linear combination of specific polynomial basis functions over the element. We would then like 
to obtain provable bounds on $J_{\min }$ by using the properties of these basis functions.

In addition to guaranteeing the geometrical validity of the curvilinear element, we are also interested in quantifying its distortion, i.e., the deformation induced by the curving. To this end, let us consider the transformation $\mathbf{X}(\mathbf{x})$ that maps straight sided elements onto curvilinear elements (see Figure 1). It is possible to write the determinant of this mapping in terms of the $\boldsymbol{\xi}$ coordinates as:

$$
\operatorname{det} \mathbf{X}_{, \mathbf{x}}=\frac{\operatorname{det} \mathbf{X}_{, \boldsymbol{\xi}}}{\operatorname{det} \mathbf{x}_{, \boldsymbol{\xi}}}=\frac{J(\boldsymbol{\xi})}{\operatorname{det} \mathbf{x}_{, \boldsymbol{\xi}}}
$$

We call $\mathbf{X}(\mathbf{x})$ the distortion mapping and its determinant $\delta(\boldsymbol{\xi}):=\operatorname{det} \mathbf{X}_{, \mathbf{x}}$ the distortion. The distortion $\delta$ should be as close to $\delta=1$ as possible in order not to degrade the quality of the straight sided element. Elements that have negative distortions are of course invalid but elements that have distortions $\delta \ll 1$ or $\delta \gg 1$ lead to some alteration of the conditioning of the finite element problem. In order to guarantee a reasonable distortion it is thus necessary to find a reliable bound on $J_{\min }$ and $J_{\max }:=\max _{\boldsymbol{\xi}} J(\boldsymbol{\xi})$ over the whole element.

Note that many different quality measures can be defined based on the Jacobian determinant $J$. For example, one could look at the Jacobian determinant divided by its average over the element instead of looking at the distortion. Obtaining bounds on $J_{\min }$ and $J_{\max }$ is thus still the main underlying challenge. Other interesting choices are presented and analyzed in $[5]$.

\section{Bounds for Second Order Planar Triangles}

We start our analysis with the particular case of second order planar triangles for which a direct computation of $J_{\min }$ is relatively easy. The determinant $J(\boldsymbol{\xi})=J(\xi, \eta)$ for a planar triangle at order $p$ is a polynomial in $\xi$ and $\eta$ of order at most $2(p-1)$. For quadratic planar triangles, $J(\xi, \eta)$ is therefore quadratic at most in $\xi$ and $\eta$.

The geometry of the six-node quadratic triangle is shown in Figure 1. Inspection reveals two types of nodes: corners (1,2 and 3$)$ and midside nodes (4,5 and 6 ). If $J_{i}$ is defined as $J(\xi, \eta)$ evaluated at node $i$, it is possible to

write the Jacobian determinant exactly as a finite element expansion whose 
coefficients are the Jacobian determinants at the nodes:

$$
\begin{aligned}
& J(\xi, \eta)= J_{1} \underbrace{(1-\xi-\eta)(1-2 \xi-2 \eta)}_{\mathcal{L}_{1}^{(2)}(\xi, \eta)}+J_{2} \underbrace{\xi(2 \xi-1)}_{\mathcal{L}_{2}^{(2)}(\xi, \eta)}+J_{3} \underbrace{\eta(2 \eta-1)}_{\mathcal{L}_{3}^{(2)}(\xi, \eta)}+ \\
& J_{4} \underbrace{4(1-\xi-\eta) \xi}_{\mathcal{L}_{4}^{(2)}(\xi, \eta)}+J_{5} \underbrace{4 \xi \eta}_{\mathcal{L}_{5}^{(2)}(\xi, \eta)}+J_{6} \underbrace{4(1-\xi-\eta) \eta}_{\mathcal{L}_{6}^{(2)}(\xi, \eta)} .
\end{aligned}
$$

In equation (4), the functions $\mathcal{L}_{i}^{(2)}(\xi, \eta)$ are the equidistant quadratic Lagrange shape functions that are commonly used in the finite element community [6].

We first show how to compute the exact minimal Jacobian determinant $J_{\min }$. Then we examine different bounds that can be provided on $J_{\min }$ by exploiting the properties of the basis used in the expansion.

\subsection{Exact Computation of $J_{\min }$}

From equation (4), the stationnary point of $J$ can be computed by solving

$$
\frac{\partial J}{\partial \xi}=\frac{\partial J}{\partial \eta}=0
$$

which leads to the following linear system of two equations and two unknowns $\xi_{\text {sta }}$ and $\eta_{\text {sta }}$ :

$$
\begin{gathered}
{\left[\begin{array}{cc}
4\left(J_{1}+J_{2}-2 J_{4}\right) & 4\left(J_{1}-J_{4}+J_{5}-J_{6}\right) \\
4\left(J_{1}-J_{4}+J_{5}-J_{6}\right) & 4\left(J_{1}+J_{3}-2 J_{6}\right)
\end{array}\right]\left(\begin{array}{c}
\xi_{\text {sta }} \\
\eta_{\text {sta }}
\end{array}\right)} \\
=\left(\begin{array}{l}
-\left(-3 J_{1}-J_{2}+4 J_{4}\right) \\
-\left(-3 J_{1}-J_{3}+4 J_{6}\right)
\end{array}\right) .
\end{gathered}
$$

Algorithm 1 allows to compute the minimal Jacobian determinant over one quadratic planar element exactly. If the minimum of the function is outside of the element, it computes the minimum on its border assuming a function $\operatorname{MINQ}(a, b, c)$ that computes

$$
\operatorname{MiNQ}(a, b, c)=\min _{x \in[0,1]} a x^{2}+b x+c .
$$

Although Algorithm 1 is quite simple, applying similar techniques for higher order elements would become extremely expensive computationally. For example, for a third order triangle, the Jacobian determinant is of order 4 and the algorithm requires the solution of a system of cubic equations; at order 4 , it requires the solution of a system of quintic equations. Instead of trying to evaluate $J_{\min }$ directly, we should try to compute (the sharpest possible) bounds in a computationally efficient manner. 


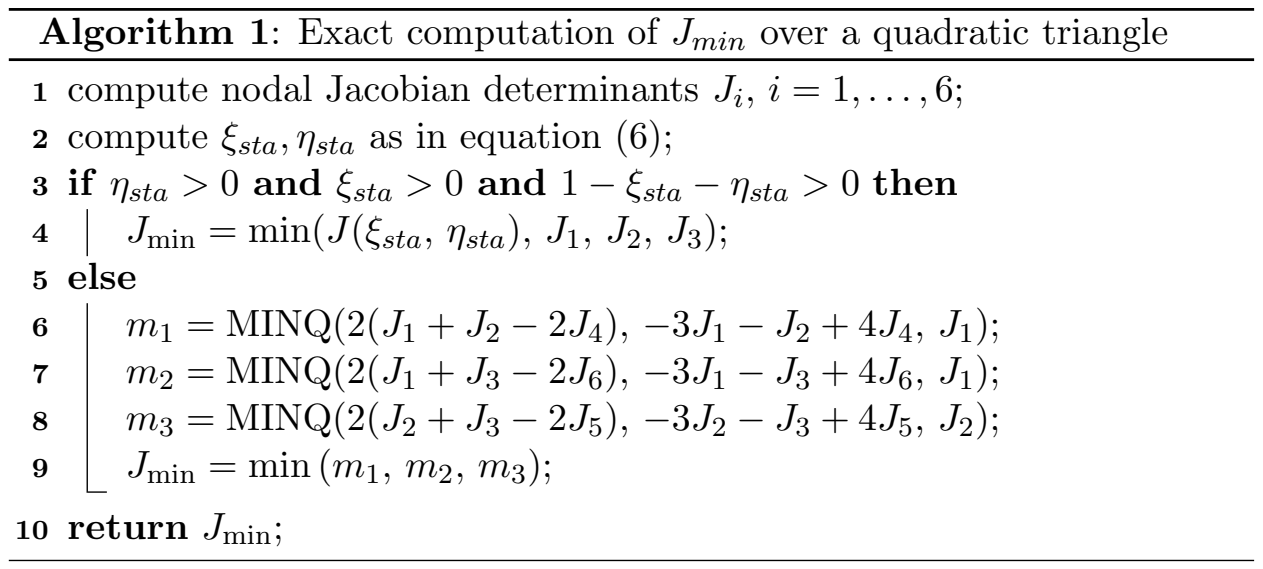

\subsection{The Principle for Computing Bounds on $J_{\min }$}

It is obvious that a necessary condition for having $J(\xi, \eta)>0$ everywhere is that $J_{i}>0, i=1, \ldots, 6$. Yet, this condition is not sufficient. The expression (4) does not give more information because the quadratic Lagrange shape functions $\mathcal{L}_{i}^{(2)}(\xi, \eta)$ change sign on the reference triangle. What polynomial basis should we chose to obtain usable bounds?

The first idea is to expand (4) into monomials, which gives:

$$
\begin{aligned}
& J(\xi, \eta)=J_{1}+\left(-3 J_{1}-J_{2}+4 J_{4}\right) \xi+\left(-3 J_{1}-J_{3}+4 J_{6}\right) \eta+ \\
& \quad 4\left(J_{1}-J_{4}+J_{5}-J_{6}\right) \xi \eta+2\left(J_{1}+J_{2}-2 J_{4}\right) \xi^{2}+2\left(J_{1}+J_{3}-2 J_{6}\right) \eta^{2} .
\end{aligned}
$$

Every monomial being positive on the reference triangle, we have now a set of sufficient conditions that can be written as

$4 J_{4} \geq 3 J_{1}+J_{2}, 4 J_{6} \geq 3 J_{1}+J_{3}, J_{1}+J_{5} \geq J_{4}+J_{6}, J_{1}+J_{2} \geq 2 J_{4}, J_{1}+J_{3} \geq 2 J_{6}$.

However these constraints do not provide a usable bound on $J_{\min }$ and break the symmetry of the expression with respect to a rotation of corner nodes.

A second idea is to expand (4) in terms of the second order hierarchical basis functions $\psi_{i}(\xi, \eta), i=1, \ldots, 6$, which are also positive on the triangle [7]:

$$
\begin{gathered}
J(\xi, \eta)=J_{1} \underbrace{(1-\xi-\eta)}_{\psi_{1}(\xi, \eta)}+J_{2} \underbrace{\xi}_{\psi_{2}(\xi, \eta)}+J_{3} \underbrace{\eta}_{\psi_{3}(\xi, \eta)}+\left(4 J_{4}-2 J_{1}-2 J_{2}\right) \underbrace{(1-\xi-\eta) \xi}_{\psi_{4}(\xi, \eta)}+ \\
\left(4 J_{5}-2 J_{3}-2 J_{2}\right) \underbrace{\xi \eta}_{\psi_{5}(\xi, \eta)}+\left(4 J_{6}-2 J_{1}-2 J_{3}\right) \underbrace{(1-\xi-\eta) \eta}_{\psi_{6}(\xi, \eta)} . \quad(9)
\end{gathered}
$$


This last expression has the right symmetry, and leads to the following validity conditions:

$J_{1} \geq 0, \quad J_{2} \geq 0, J_{3} \geq 0,4 J_{4} \geq 2 J_{1}+2 J_{2}, 4 J_{5} \geq 2 J_{2}+2 J_{3}, 4 J_{6} \geq 2 J_{3}+2 J_{1}$.

$J(\xi, \eta)$ is a degree two polynomial, therefore it has an expression in the $\left(\Psi_{i}\right)$ basis. Let $K_{i}$ denote the coefficients in this basis. Writing $J(\xi, \eta):=$ $\sum_{i=1}^{6} \psi_{i}(\xi, \eta) K_{i}$, we have

$\min _{\xi, \eta} J(\xi, \eta)=\min _{\xi, \eta}\left(\sum_{i} \psi_{i}(\xi, \eta) K_{i}\right) \geq \min _{\xi, \eta}\left(\sum_{i} \psi_{i}(\xi, \eta)\right) \min _{i} K_{i}=\min _{i} K_{i}$,

because $\sum_{i} \psi_{i}=1+\xi+\eta-\xi^{2}-\eta^{2}-\xi \eta$ has its minimum on the corner nodes (where its value is equal to 1 ). And since $K_{i}, i=1, \ldots, 3$ are values of the Jacobian determinant (at the three corners), they form an upper bound on it. Thus, expansion (9) leads to the following estimate for the minimum of the Jacobian determinant over the triangle:

$$
\begin{aligned}
J_{\min } & \geq \min \left\{J_{1}, J_{2}, J_{3}, 4 J_{4}-2 J_{1}-2 J_{2}, 4 J_{5}-2 J_{2}-2 J_{3}, 4 J_{6}-2 J_{3}-2 J_{1}\right\} \\
& \leq \min \left\{J_{1}, J_{2}, J_{3}\right\} .
\end{aligned}
$$

It is easy to see that the estimate is however of very poor quality: for an element that has a constant and positive $J,(11)$ simply tells us that $J_{\min } \geq 0$.

In order to find a sharper estimate, instead of the hierarchical quadratic functions $\psi_{i}(\xi, \eta)$, we can use the quadratic triangular Bézier functions $\mathcal{B}_{2}^{(2)}(\xi, \eta)[8]:$

$$
\begin{gathered}
J(\xi, \eta)=J_{1} \underbrace{(1-\xi-\eta)^{2}}_{\mathcal{B}_{1}^{(2)}(\xi, \eta)}+J_{2} \underbrace{\xi^{2}}_{\mathcal{B}_{2}^{(2)}(\xi, \eta)}+J_{3} \underbrace{\eta^{2}}_{\mathcal{B}_{3}^{(2)}(\xi, \eta)}+ \\
\left(2 J_{4}-\frac{1}{2}\left(J_{2}+J_{1}\right)\right) \underbrace{2 \xi(1-\xi-\eta)}_{\mathcal{B}_{4}^{(2)}(\xi, \eta)}+\left(2 J_{5}-\frac{1}{2}\left(J_{3}+J_{2}\right)\right) \underbrace{2 \xi \eta}_{\mathcal{B}_{5}^{(2)}(\xi, \eta)}+ \\
\left(2 J_{6}-\frac{1}{2}\left(J_{1}+J_{3}\right)\right) \underbrace{2 \eta(1-\xi-\eta)}_{\mathcal{B}_{6}^{(2)}(\xi, \eta)}
\end{gathered}
$$

Since $\sum_{i=1}^{6} \mathcal{B}_{i}^{(2)}(\xi, \eta)=1$ and $\mathcal{B}_{i}^{(2)}(\xi, \eta) \geq 0$, we obtain the following esti- 
mate

$$
\begin{aligned}
J_{\min } & \geq \min \left\{J_{1}, J_{2}, J_{3}, 2 J_{4}-\frac{J_{1}+J_{2}}{2}, 2 J_{5}-\frac{J_{2}+J_{3}}{2}, 2 J_{6}-\frac{J_{3}+J_{1}}{2}\right\} \\
& \leq \min \left\{J_{1}, J_{2}, J_{3}\right\} .
\end{aligned}
$$

One can show that this estimate is always better than the one using the hierarchical basis. It provides two conditions on the geometrical validity of the triangle: a sufficient condition (if $\min \left\{J_{1}, J_{2}, J_{3}, 2 J_{4}\right.$ $\left.\frac{J_{1}+J_{2}}{2}, 2 J_{5}-\frac{J_{2}+J_{3}}{2}, 2 J_{6}-\frac{J_{3}+J_{1}}{2}\right\}>0$, the element is valid) and a necessary condition (if $\min \left\{J_{1}, J_{2}, J_{3}\right\}<0$, the element is invalid). However, these two conditions are sometimes insufficient to determine the validity of the element, as the bound (13) is often not sharp enough (having $\min \left\{2 J_{4}-\frac{J_{1}+J_{2}}{2}, 2 J_{5}-\frac{J_{2}+J_{3}}{2}, 2 J_{6}-\frac{J_{3}+J_{1}}{2}\right\}<0$ does not imply that the element is invalid).

A sharp necessary and sufficient condition on the geometrical validity of an element can be achieved in a general way by refining the Bézier estimate adaptively so as to achieve any prescribed tolerance - and thus provide bounds as sharp as necessary for a given application.

\section{Adaptive Bounds for Arbitrary Curvilinear Finite Elements}

In order to explain the adaptive bound computation let us first focus on the one-dimensional case, for "line" finite elements. Since Bézier functions can be generated for all types of common elements (triangles, quadrangles, tetrehedra, hexahedra and prisms), the generalization to $2 \mathrm{D}$ and $3 \mathrm{D}$ elements will be straightforward.

\subsection{The One-Dimensional Case}

In 1D the Bézier functions are the Bernstein polynomials:

$$
\mathcal{B}_{k}^{(n)}(\xi)=\left(\begin{array}{l}
n \\
k
\end{array}\right)(1-\xi)^{n-k} \xi^{k} \quad(\xi \in[0,1] ; k=0, \ldots, n)
$$

where $\left(\begin{array}{l}n \\ k\end{array}\right)=\frac{n !}{n !(n-k) !}$ is the binomial coefficient. The Bézier interpolation requires $n+1$ control values $b_{i}$. We have

$$
J(\xi)=\sum_{k=0}^{N_{n}} \mathcal{B}_{k}^{(n)}(\xi) b_{k} .
$$

Bernstein-Bézier functions have the nice following properties : (i) they form a partition of unity which means that $\sum_{k=0}^{n} \mathcal{B}_{k}^{(n)}(\xi)=1$ for all $\xi \in[0,1]$ and 
(ii) they are positive which means that $\mathcal{B}_{k}^{(n)}(\xi) \geq 0$ for all $\xi \in[0,1]$. This leads to the well known property of Bézier interpolations:

$$
\min _{\xi \in[0,1]} J(\xi) \geq b_{\min }=\min _{i} b_{i} \text { and } \max _{\xi \in[0,1]} J(\xi) \leq b_{\max }=\max _{i} b_{i} .
$$

Moreover, the control values related to the corner nodes of the element are equal to the values of the interpolated function. In what follows we assume that these "corner" control values are always ordered at the $K_{f}$ first indices. We then have

$$
\min _{\xi \in[0,1]} J(\xi) \leq \min _{i<K_{f}} b_{i} \text { and } \max _{\xi \in[0,1]} J(\xi) \geq \max _{i<K_{f}} b_{i} .
$$

Since Lagrange and Bézier functions span the same function space, computation of the Bézier values $b_{i}$ from the nodal values $J_{i}$ (and convertly) is done by a transformation matrix. The tranformation matrix $\boldsymbol{T}_{\mathcal{B} \rightarrow \mathcal{L}}^{(n)}$, which computes nodal values from control values, is created by evaluating Bézier functions at sampling points:

$$
\boldsymbol{T}_{\mathcal{B} \rightarrow \mathcal{L}}^{(n)}=\left[\begin{array}{ccc}
\mathcal{B}_{0}^{(n)}\left(\xi_{0}\right) & \ldots & \mathcal{B}_{n}^{(n)}\left(\xi_{0}\right) \\
\mathcal{B}_{0}^{(n)}\left(\xi_{1}\right) & \ldots & \mathcal{B}_{n}^{(n)}\left(\xi_{1}\right) \\
\vdots & \ddots & \vdots \\
\mathcal{B}_{0}^{(n)}\left(\xi_{n}\right) & \ldots & \mathcal{B}_{n}^{(n)}\left(\xi_{n}\right)
\end{array}\right]
$$

Those sampling points are taken uniformly, i.e. at the location of the nodes of the element of order $n$. The inverse transformation is $\boldsymbol{T}_{\mathcal{L} \rightarrow \mathcal{B}}^{(n)}=\boldsymbol{T}_{\mathcal{B} \rightarrow \mathcal{L}}^{(n)}{ }^{-1}$ and from the expression of the interpolation of the Jacobian determinant (15), we can write

$$
\begin{aligned}
J & =\boldsymbol{T}_{\mathcal{B} \rightarrow \mathcal{L}}^{(n)} B \\
B & =\boldsymbol{T}_{\mathcal{L} \rightarrow \mathcal{B}}^{(n)} J,
\end{aligned}
$$

where $B$ and $J$ are the vectors containing respectively the $b_{i}$ 's and the $J_{i}$ 's.

\subsection{Adaptive Subdivision}

Let us assume that the domain $[0,1]$ is subdivided into $Q$ parts. The interpolation $J^{[q]}\left(\xi^{[q]}\right)$ on the $q^{\text {th }}$ subdomain $[a, b](0 \leq a<b \leq 1)$ must verify

$$
J^{[q]}\left(\xi^{[q]}\right)=\sum_{k=0}^{N_{n}} \mathcal{B}_{k}^{(n)}\left(\xi^{[q]}\right) b_{k}^{[q]}=\sum_{k=0}^{N_{n}} \mathcal{B}_{k}^{(n)}\left(\xi\left(\xi^{[q]}\right)\right) b_{k} \quad\left(\xi^{[q]} \in[0,1]\right),
$$


with $\xi\left(\xi^{[q]}\right)=a+(b-a) \xi^{[q]}$.

Considering the nodes $\xi_{k}^{[q]}$ such that $\xi_{k}^{[q]}=\xi_{k}(k=0, \ldots, n)$ (i.e., such that they are ordered like the sampling points), the expression (19) reads

$$
\boldsymbol{T}_{\mathcal{B} \rightarrow \mathcal{L}}^{(n)} B^{[q]}=\left[\begin{array}{ccc}
\mathcal{B}_{0}^{(n)}\left(a+(b-a) \xi_{0}\right) & \ldots & \mathcal{B}_{n}^{(n)}\left(a+(b-a) \xi_{0}\right) \\
\mathcal{B}_{0}^{(n)}\left(a+(b-a) \xi_{1}\right) & \ldots & \mathcal{B}_{n}^{(n)}\left(a+(b-a) \xi_{1}\right) \\
\vdots & \ddots & \vdots \\
\mathcal{B}_{0}^{(n)}\left(a+(b-a) \xi_{n}\right) & \ldots & \mathcal{B}_{n}^{(n)}\left(a+(b-a) \xi_{n}\right)
\end{array}\right] B=\boldsymbol{T}_{\mathcal{B} \rightarrow \mathcal{L}}^{(n)}{ }^{[q]} B
$$

where $B^{[q]}$ is the vector containing the control values of the $q^{\text {th }}$ subdomain. This implies that

$$
B^{[q]}=\left[\boldsymbol{T}_{\mathcal{L} \rightarrow \mathcal{B}}^{(n)} \boldsymbol{T}_{\mathcal{B} \rightarrow \mathcal{L}}^{(n)}{ }^{[q]}\right] B=\boldsymbol{M}^{[q]} B
$$

Each set of new control values bounds the Jacobian determinant on its own subdomain and we have:

$$
b_{\min }^{\prime}=\min _{i, q} b_{i}^{[q]} \leq J_{\min } \leq \min _{i<K_{f}, q} b_{i}^{[q]}
$$

and

$$
\max _{i<K_{f}, q} b_{i}^{[q]} \leq J_{\max } \leq b_{\max }^{\prime}=\max _{i, q} b_{i}^{[q]}
$$

If an estimate is not sufficiently sharp, we can thus simply subdivide the appropriate parts of the element. This leads to a simple adaptive algorithm, exemplified in Figure 2. In this particular case the original estimate (16)-(17) is not sharp enough $\left(J_{\min } \in[-3,1]\right)$. After one subdivision, the Jacobian determinant is proved to be positive on the second subdomain. The first subdomain is thus subdivided once more, which proves the validity. In practice, as will be seen in Section 5, a few levels of refinement lead to the desired accuracy. The subdivision has quadratic speed of convergence [9, 10].

Note that in a practical implementation (in finite precision arithmetic) we must take care of a tricky situation. If the minimum of the Jacobian determinant is too close to zero but positive, then the upper bound is positive while the lower bound might never get positive. In order to avoid this situation, we limit the number of consecutive subdivisions that can be applied. The undetermined elements are then considered as invalid. Another way of getting rid of this issue is to relax the condition of rejection as explained in section 4.4. 

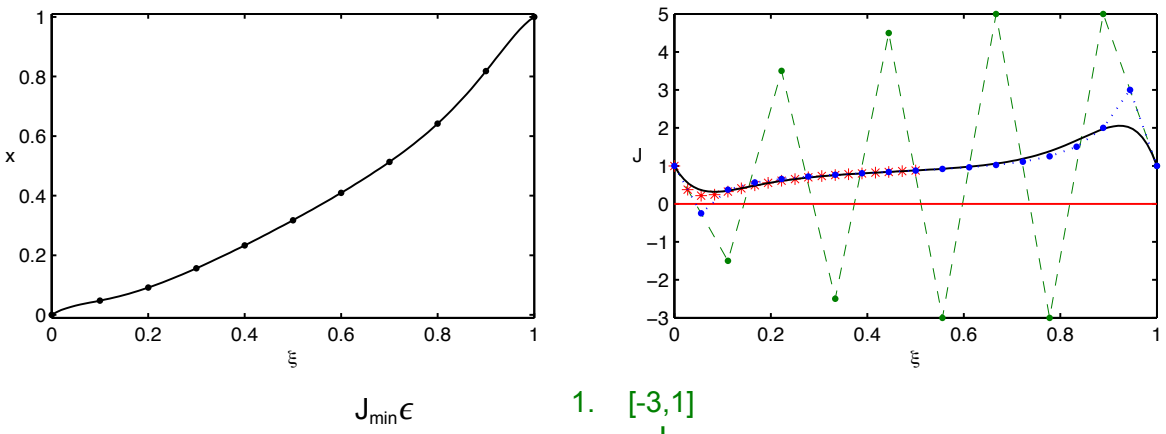

1. $[-3,1]$

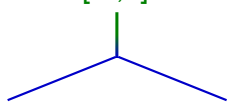

2. $[-0.25,0.88]$

$[0.88,0.88]$

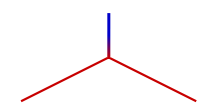

3. $[0.22,0.65][0.65,0.65]$

Figure 2: Top left: One-dimensional element mapping $x(\xi)$. Top right: Exact Jacobian determinant $J(\xi)$ (black), control values on the original control points (green) and two adaptive subdivisions (blue and red). Bottom: Estimates of $J_{\min }$ at each step in the adaptive subdivision process. 


\subsection{Extension to Higher Dimensions}

The extension of the method to higher dimensions is straightforward, provided that Bézier functions can be generated and that a subdivision scheme is available. Jacobian determinants $J$ are polynomials of $\xi, \eta$ in 2D and of $\xi, \eta, \zeta$ in $3 \mathrm{D}$.

For high order triangles, the Bézier triangular polynomials are defined as

$$
\mathcal{T}_{i, j}^{(p)}(\xi, \eta)=\left(\begin{array}{c}
p \\
i
\end{array}\right)\left(\begin{array}{c}
p-i \\
j
\end{array}\right) \xi^{i} \eta^{j}(1-\xi-\eta)^{p-i-j} \quad(i+j \leq p) .
$$

It is possible to interpolate any polynomial function of order at most $p$ on the unit triangle $\xi>0, \eta>0, \xi+\eta<1$ as an expansion into Bézier triangular polynomials. Recalling that, for a triangle at order $p$, its Jacobian determinant $J(\xi, \eta)$ is a polynomial in $\xi$ and $\eta$ at order at most $n=2(p-1)$, we can write

$$
J(\xi, \eta)=\sum_{i+j \leq n} b_{i j} \mathcal{T}_{i, j}^{(n)}(\xi, \eta) .
$$

It is also possible to compute $J$ in terms of Lagrange polynomials

$$
J(\xi, \eta)=\sum_{i} J_{i} \mathcal{L}_{i}^{(n)}(\xi, \eta)
$$

where the $J_{i}$ are the Jacobian determinants calculated at Lagrange points. It is then easy to find a transformation matrix $T_{\mathcal{L B}}^{n}$ such that

$$
B=T_{\mathcal{L B}}^{n} J
$$

where $B$ and $J$ are the vectors containing respectively the control values of the Jacobian determinant $b_{i j}$ and the $J_{i}$ 's. As an example, for quadratic triangles we obtain

$$
T_{\mathcal{L B}}^{2}=\left(\begin{array}{rrrrrr}
1 & 0 & 0 & 0 & 0 & 0 \\
0 & 1 & 0 & 0 & 0 & 0 \\
0 & 0 & 1 & 0 & 0 & 0 \\
-1 / 2 & -1 / 2 & 0 & 2 & 0 & 0 \\
0 & -1 / 2 & -1 / 2 & 0 & 2 & 0 \\
-1 / 2 & 0 & -1 / 2 & 0 & 0 & 2
\end{array}\right)
$$

which directly provides the estimate (13).

Other element shapes can be treated similarly. For quadrangles, tetrahedra, prisms and hexahedra, the Bézier are functions respectively:

$$
\mathcal{Q}_{i, j}^{(p)}(\xi, \eta)=\mathcal{B}_{i}^{(p)}(\xi) \mathcal{B}_{j}^{(p)}(\eta) \quad(i \leq p, j \leq p),
$$




\begin{tabular}{r|c|c}
\hline & Order $(n)$ of $J$ & Number of coefficients \\
\hline Line & $p-1$ & $n+1$ \\
Triangle & $2(p-1)$ & $(n+1)(n+2) / 2$ \\
Quadrangle & $2 p-1$ & $(n+1)^{2}$ \\
Tetrahedron & $3(p-1)$ & $(n+1)(n+2)(n+3) / 6$ \\
Prism & $3 p-1$ & $(n+1)^{2}(n+2) / 2$ \\
Hexahedron & $3 p-1$ & $(n+1)^{3}$ \\
\hline
\end{tabular}

Table 1: Order of the Jacobian determinant and number of coefficients in the expansion for an element of order $p$.

$$
\begin{gathered}
\mathcal{T}_{i, j, k}^{(p)}(\xi, \eta, \zeta)=\left(\begin{array}{c}
p \\
i
\end{array}\right)\left(\begin{array}{c}
p-i \\
j
\end{array}\right)\left(\begin{array}{c}
p-i-j \\
k
\end{array}\right) \xi^{i} \eta^{j} \zeta^{k}(1-\xi-\eta-\zeta)^{p-i-j-k} \\
(i+j+k \leq p), \\
\mathcal{P}_{i, j, k}^{(p)}(\xi, \eta, \zeta)=\mathcal{T}_{i, j}^{(p)}(\xi, \eta) \mathcal{B}_{k}^{(p)}(\zeta) \quad(i+j \leq p, k \leq p)
\end{gathered}
$$

and

$$
\mathcal{H}_{i, j, k}^{(p)}(\xi, \eta, \zeta)=\mathcal{B}_{i}^{(p)}(\xi) \mathcal{B}_{j}^{(p)}(\eta) \mathcal{B}_{k}^{(p)}(\zeta) \quad(i \leq p, j \leq p, k \leq p) .
$$

Matrices of change of coordinates can then be computed inline for every polynomial order, and bounds of Jacobian determinants computed accordingly. Table 1 summarizes the order of the Jacobian determinant and the number of coefficients in its expansion for all common element types. In all cases the subdivision scheme works exactly in the same way as for lines. Figure 3 shows the first level of subdivision for a third-order triangle.

\subsection{Implementation}

As mentioned in Section 2, the bounds on the Jacobian determinant can be used to either make the distinction between valid and invalid elements with respect to a condition on $J_{\mathrm{min}}$, or to measure the quality of the elements by systematically computing $J_{\min }$ and $J_{\max }$ with a prescribed accuracy.

In both cases the same operations are executed on each element. First, the Jacobian determinant is sampled on a determined number of points $N_{s}$, equal to the dimension of the Jacobian determinant space, and so to the number of Bézier functions. Second, Bézier values are computed. Then adaptive subdivision is executed if necessary. Algorithms 2 and 3 show in pseudo-code the algorithm used to determine whether the Jacobian determinant of the element is everywhere positive or not. 

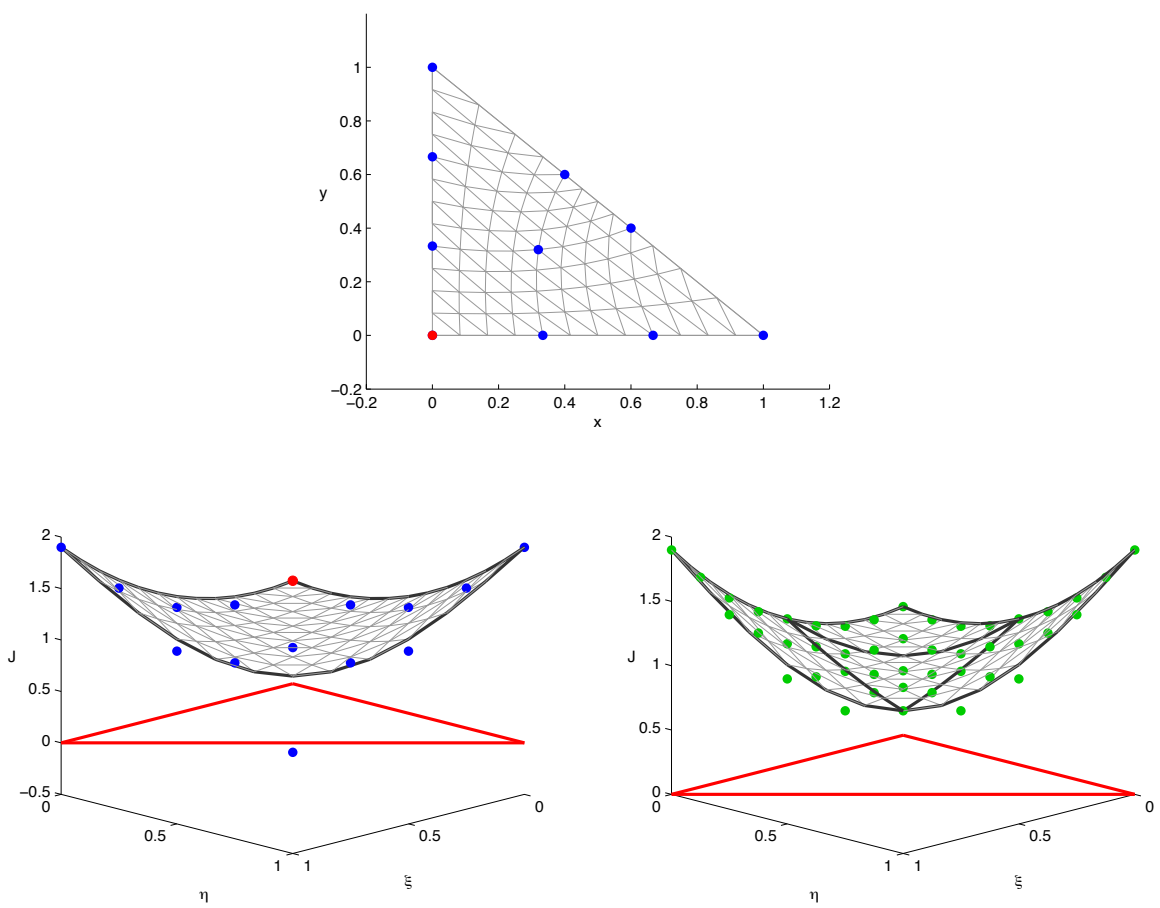

Figure 3: Top: third-order planar triangle. Bottom left: exact Jacobian determinant and control values (dots) on the original control points; the validity of the element cannot be asserted. Bottom right: exact Jacobian determinant and control values (dots) after one subdivision; the element is provably correct.

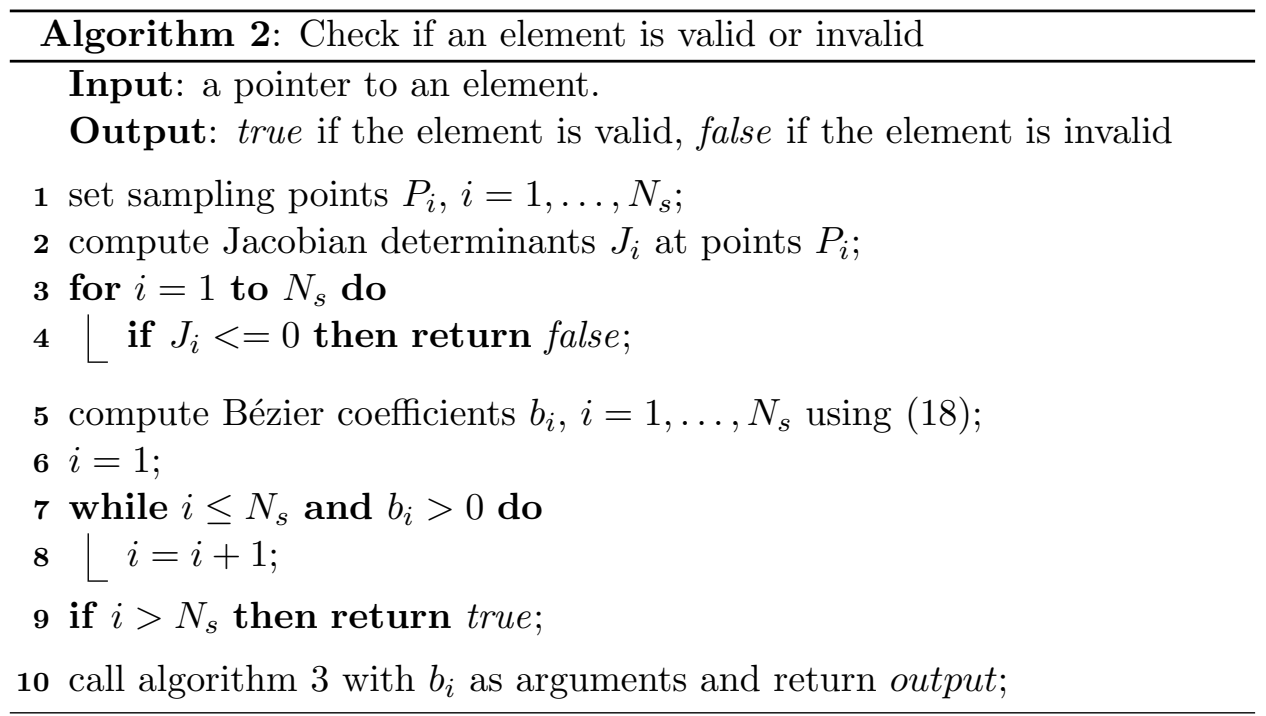




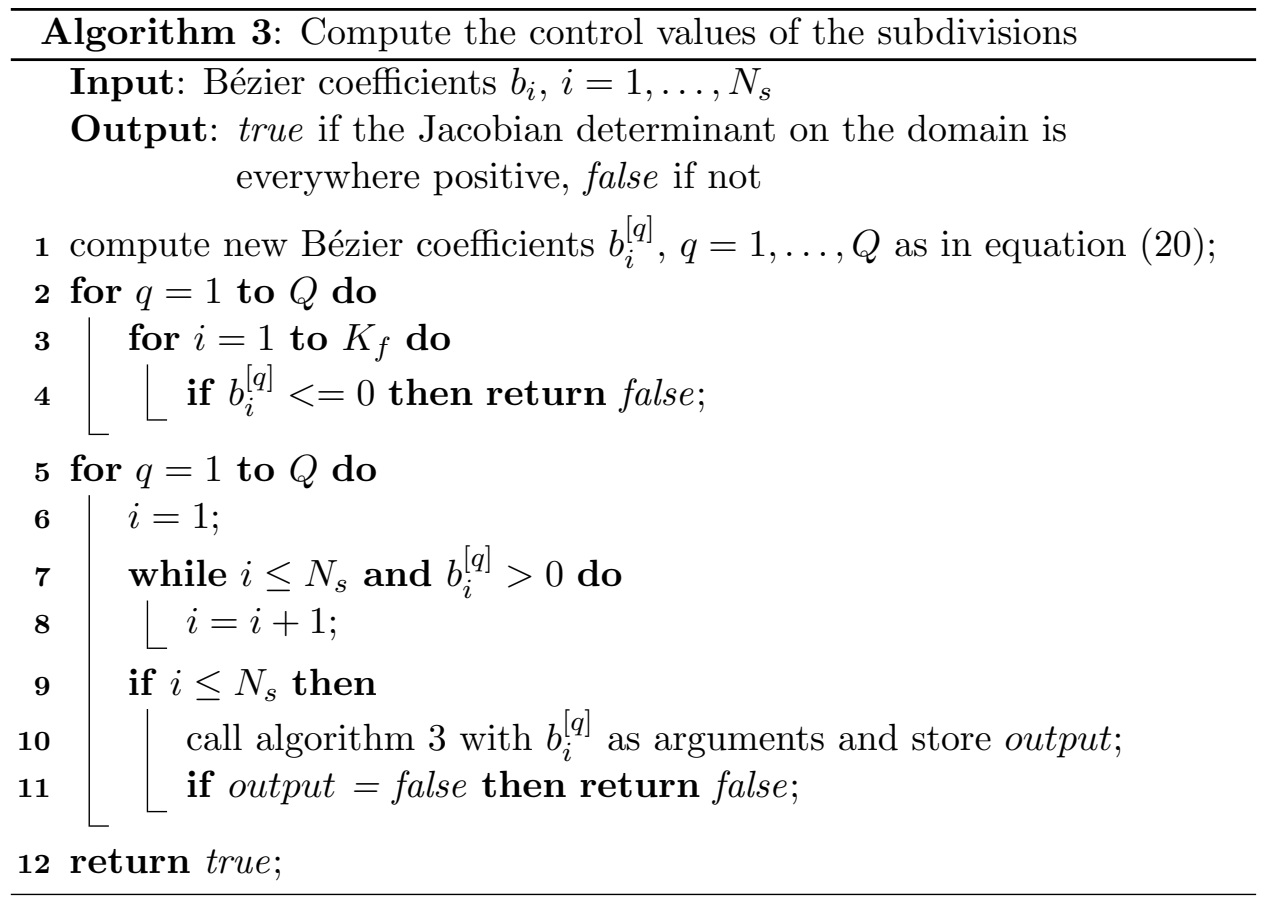

Algorithm 3 could be further improved by optimzing the loop on line 5 , by first selecting $q$ for which we have the best chance to have a negative Jacobian determinant (line 4, algo 3 ). In practice this improvement is not significant since the only case for which we can save calculation is for invalid elements - and the proportion of them which require subdivision in order to be detected is usually small (about $3 \%$ for the mesh depicted in Figure 4). Note that we may also want to find, for example, all the elements for which the Jacobian determinant is somewhere smaller than $20 \%$ of its average. We then just have to compute this average and replace the related lines ( 4 and 7 for algorithm 2).

Another possible improvement is to relax the condition of rejection. We could accept elements for which all control values are positive but reject an element as soon as we find a Jacobian determinant smaller than a defined percent of the average Jacobian determinant. The computational gain can be significant, since elements that were classified as good and which needed a lot of subdivisions (and have a Jacobian determinant close to zero) will be instead rapidly be detected as invalid.

More interestingly, the computation of sampled Jacobian determinants and the computation of Bézier control values in algorithm 2 can easily be 
executed for a whole groups of elements at the same time. This allows to use efficient BLAS 3 (matrix-matrix product) functions, which significantly speeds up the computations.

The algorithm used for all the tests in the next section is implemented in the open source mesh generator Gmsh [4] as the AnalyseCurvedMesh plugin.

\section{Numerical Results}

We start by comparing the new adaptive computation of bounds on Jacobian determinants with the brute-force sampling of the Jacobian determinant for the detection of invalid high-order triangles.

The points at which we sample the Jacobian determinant for the bruteforce method are taken as the nodes of an element of order $k$, for $k=$ $1, \ldots, 65$, leading to a number of sampling points comprised between 3 and 2211. In order to make the comparison as fair as possible, we have implemented the brute-force computation as efficiently as possible, i.e., for $k(>n)$ sufficiently large we sample the Jacobian determinant on the points computed for an element at order $n$ (the order of the Jacobian determinant) and then compute the desired Jacobian determinant values by a matrixvector product, just like in our own adaptive method.

We consider the two-dimensional microstructure with oval holes depicted in Figure 4, meshed with 82,009 sixth-order triangles. In this mesh 38,978 triangles are curved, and 8,112 are invalid. The new algorithm successfully detects all the 8,112 invalid elements in $2.146 \mathrm{~s}$ on a $2.4 \mathrm{GHz}$ Core 2 Duo laptop computer ${ }^{1}$. Some elements needed as much as 7 levels of subdivisions in order to be classified: see Table 2. The brute-force approach required 1,596 sample points per triangle $(k=55)$ in order to detect all the invalid elements, and took more than 7 times longer. But far worse, increasing the number of sampling points beyond 1,596 can actually lead to a decreased accuracy of the prediction, as shown in Figure 5.

Let us now examine the use of the adaptive bounds in the curvilinear meshing algorithm. We consider the boundary layer mesh of the B-Spline CAD model of a tri-part wing: see Figure 6. The cubic triangular mesh is generated as follows. We first generate a straight-sided mesh (Figure $6 /(\mathrm{a})$ ). Then, every mesh edge that is classified on a model edge is curved by snapping their center vertices on the model edge. High order nodes are

\footnotetext{
${ }^{1}$ Note that for completeness the algorithm also analyzes straight-sided elements, which is unnecessary in practice.
} 

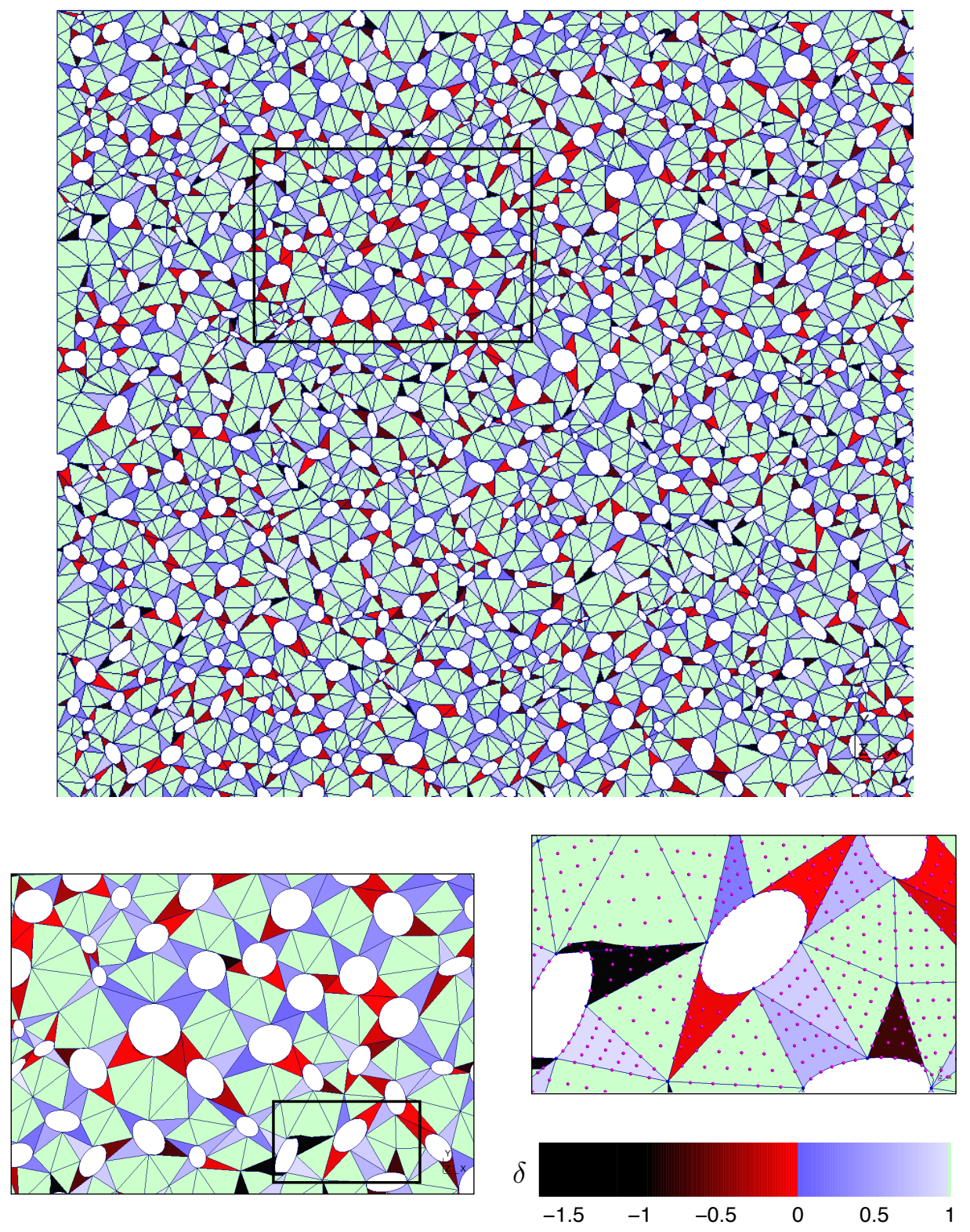

Figure 4: Two-dimensional mesh with sixth order triangles; $47.5 \%$ of the elements are curved. The straight elements are in green and the curved ones are colored in function of the minimum of the distortion. The valid elements $\left(\delta_{\min }>0\right)$ are colored in blue. The invalid ones are colored in red if $\delta_{\min }$ is near 0 and in black if $\delta_{\min }<-1.5$. 


\begin{tabular}{|c|c|c|c|c|c|}
\hline & \multicolumn{3}{|c|}{ Curved Element Classification } & \multirow{2}{*}{$\begin{array}{l}\text { \# elements } \\
\text { analysed at } \\
\text { given stage }\end{array}$} & \multirow{2}{*}{$\begin{array}{l}\text { CPU time for } \\
\text { given stage }[\mathrm{s}]\end{array}$} \\
\hline & $\begin{array}{c}\text { Valid } \\
\text { elements }\end{array}$ & $\begin{array}{c}\text { Invalid } \\
\text { elements }\end{array}$ & $\begin{array}{c}\text { Undertermined } \\
\text { elements }\end{array}$ & & \\
\hline First stage & 29,715 & 8,039 & 1,224 & 38,978 & 1.865 \\
\hline 1 subdiv. & +787 & +0 & 437 & 1,224 & $1.16 \mathrm{e}-1$ \\
\hline 2 subdiv. & +285 & +17 & 135 & 437 & $8.40 \mathrm{e}-2$ \\
\hline 3 subdiv. & +56 & +15 & 64 & 135 & $4.02 \mathrm{e}-2$ \\
\hline 4 subdiv. & +16 & +22 & 26 & 64 & $2.40 \mathrm{e}-2$ \\
\hline 5 subdiv. & +5 & +15 & 6 & 26 & $1.10 \mathrm{e}-2$ \\
\hline 6 subdiv. & +1 & +2 & 3 & 6 & $4.34 \mathrm{e}-3$ \\
\hline 7 subdiv. & +1 & +2 & 0 & 3 & $1.47 \mathrm{e}-3$ \\
\hline Subtotal & 30,866 & 8,112 & & & \multirow{2}{*}{2.146} \\
\hline Total & \multicolumn{2}{|c|}{38,978} & & & \\
\hline
\end{tabular}

Table 2: On the left, number of curved elements detected as valid or invalid at each stage of the adaptive algorithm; At the first stage, 8,309 elements can be classified as invalid due to a negative value of at least one of the 66 sampling points. Then Bézier coefficients are computed and 29,715 elements are classified as valid because those coefficients are positive. The 1,224 (3.14\%) remaining elements need to be subdivided adaptively. On the right, computation time; most of the time is spent on sampling the Jacobian determinant and computing the first Bézier coefficients.

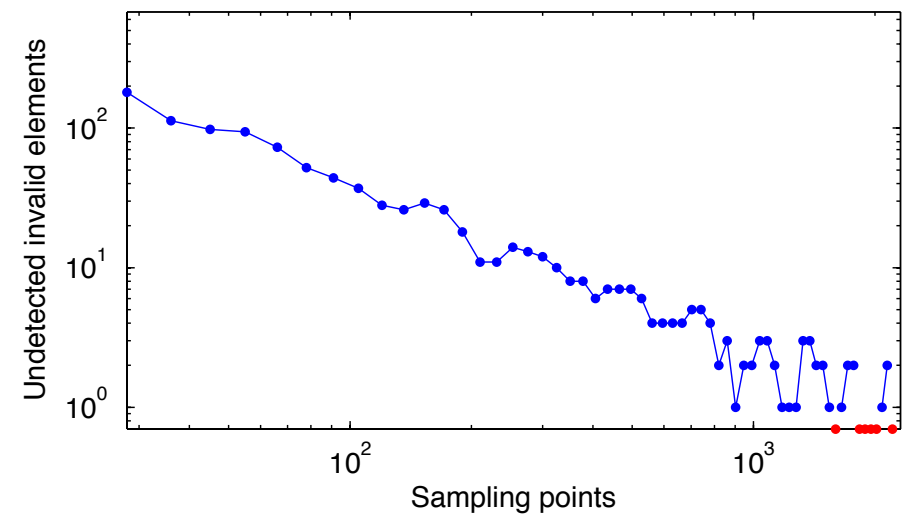

Figure 5: Number of undetected invalid elements using brute-force sampling of the Jacobian determinant. The five red data points correspond to the correct result, i.e., when no invalid triangle is left undetected. 
then inserted on every edge and in the middle of every face (Figure 6/(b)). This simple procedure does not guarantee that the final mesh is valid. In our case, 66 elements are invalid. Then, an optimization is applied globally (Figure 6/(c)). The final curvilinear mesh contains about $31 \%$ of curved elements. During the meshing process, the adaptive bound computation allowed to detect all invalid elements (the worst minimum of the distortion that was observed was $\delta_{\min }=-27.72$ ). After optimization, the final mesh is composed of elements that have $\delta_{\min }>0.64$.

Finally, a 3D mesh is considered. The CAD model of an A319 plane is meshed with 168,884 p3 tetrahedra (see figure 7 and 8). Without executing any optimization, 76 elements are invalid. The new algorithm detects them in $9.88 \mathrm{~s}$ on a $2.4 \mathrm{GHz}$ Core 2 Duo laptop computer. The worst elements in term of their Jacobian determinant are located around leading edges, where the curvature is the most important. 999 elements have $\delta_{\min } \leq .7$ and the worst distortion is $\delta_{\min }=-7.74$.

\section{Conclusions and Perspectives}

In this paper we presented a way to compute accurate bounds on Jacobian determinants of curvilinear finite elements, based on the efficient expansion of these Jacobian determinants in terms of Bézier functions.

The overall idea can be summarized as follows:

1. The Jacobian determinant of a polynomial element is also a polynomial (of higher order).

2. The Bézier polynomial basis satisfies the convex hull property, which means that any polynomial expressed in this basis is bounded by the values at the control nodes.

3. By a change of polynomial basis (e.g. from Lagrange to Bézier), one naturally gets the bounds of the Jacobian determinant.

4. If the bounds are not accurate enough, one can subdivide the element, and once again, with a change of polynomial basis, obtain a more accurate bound in each sub-element (and so on and so forth until enough precision is reached).

The proposed algorithm can either be used to determine the validity or invalidity of curved elements, or provide an efficient way to measure their distortion. Triangles, quadrangles, tetrahedra, prisms and hexahedra can be analyzed using the same algorithm, which is available in the open source mesh generator Gmsh. Numerical tests show that the method is 


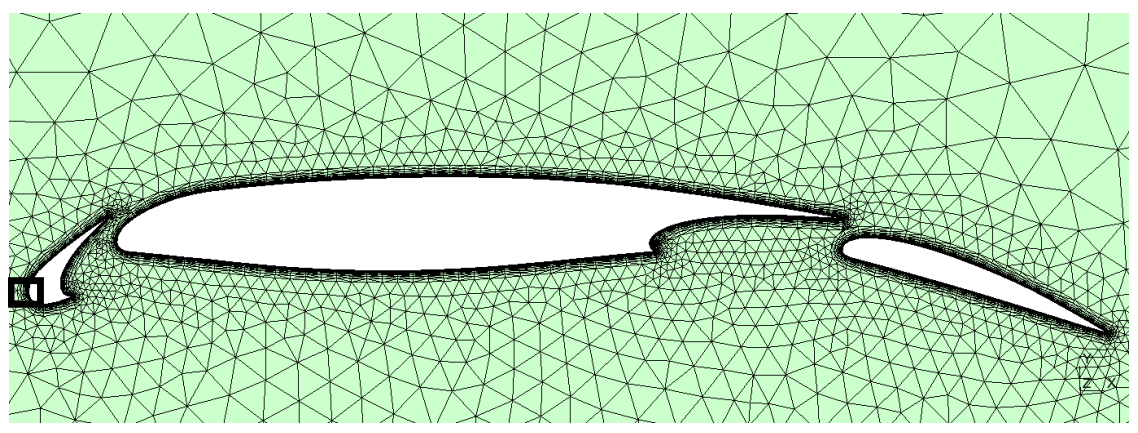

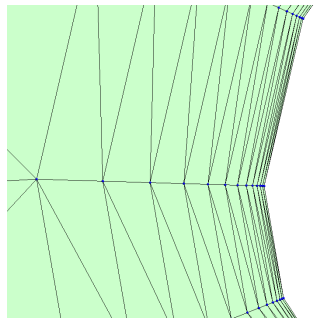

(a) Straight-sided mesh

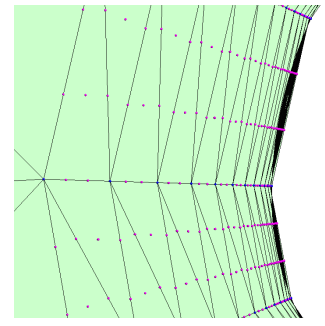

(b) Raw curvilinear mesh

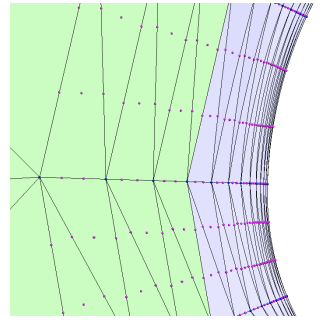

(c) After optimization

$\delta$
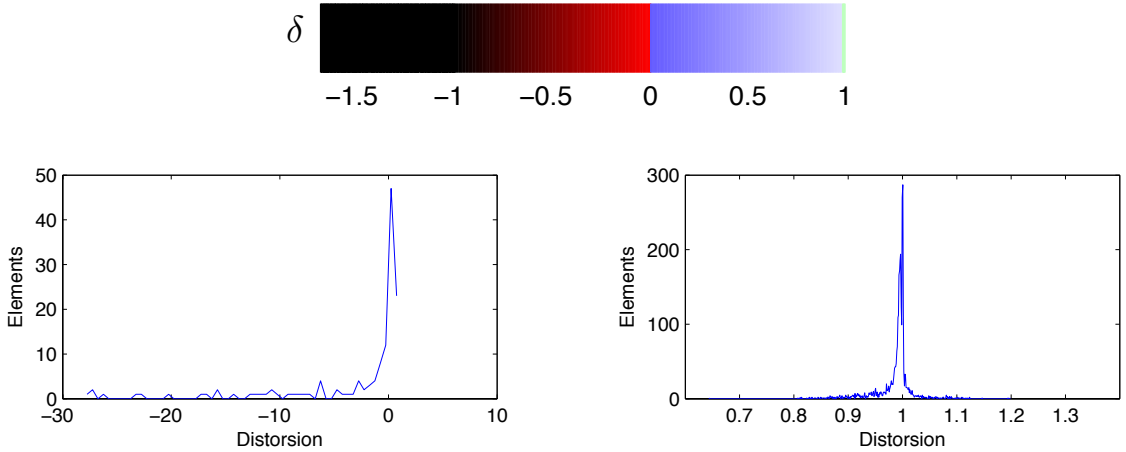

(d) Distortion before and after optimization

Figure 6: Distortion of the curvilinear mesh of a wing (p3 triangles) before and after optimization. 


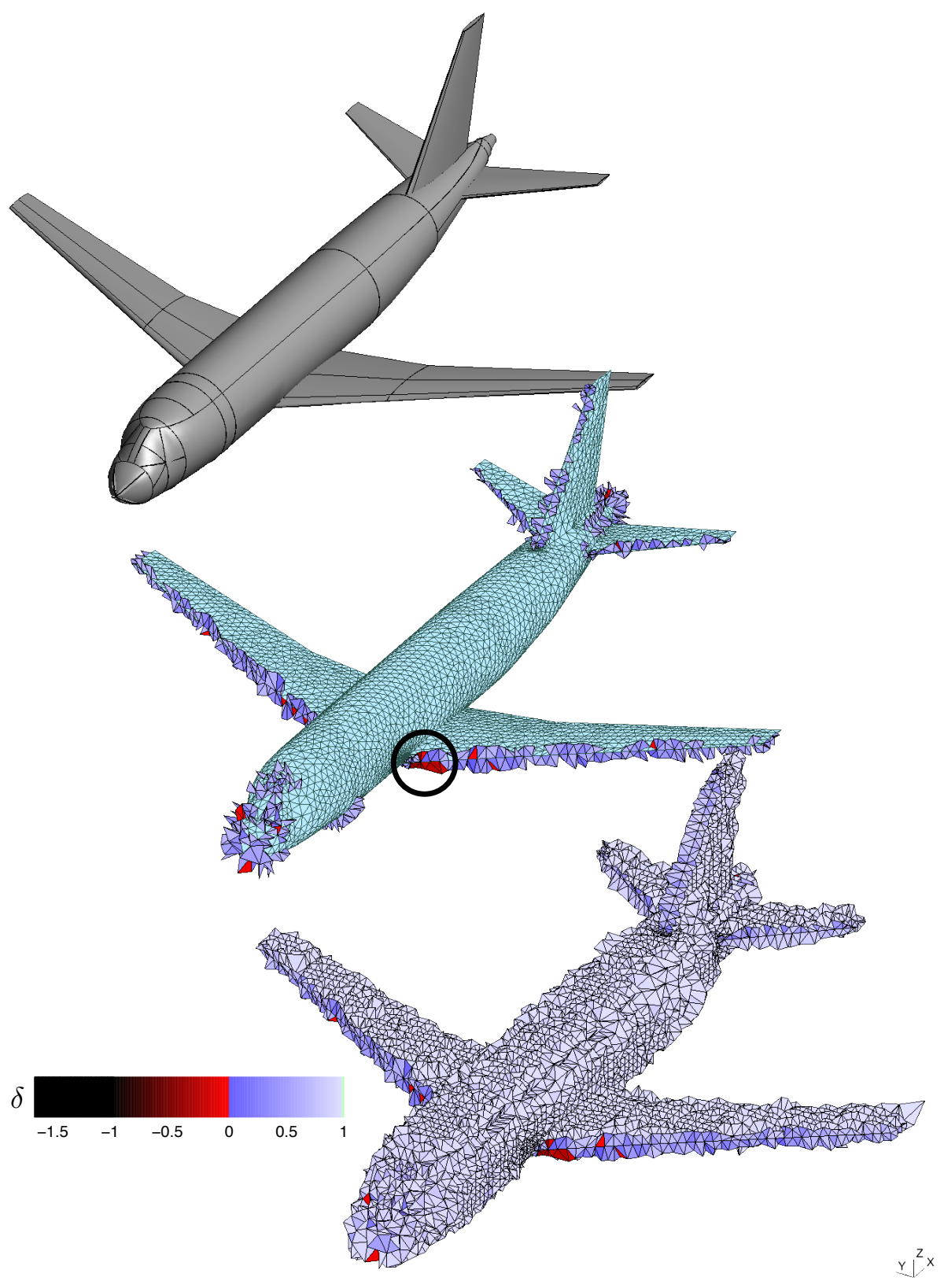

Figure 7: The geometry of a A319 plane is meshed with p3 tetrahedron without executing optimization. The curved elements are all shown in the figure at the bottom. For the figure at the center, only elements for which $\delta \leq 0.7$ are shown. On 168,884 elements, 24,691 are curved and 76 are invalid. 

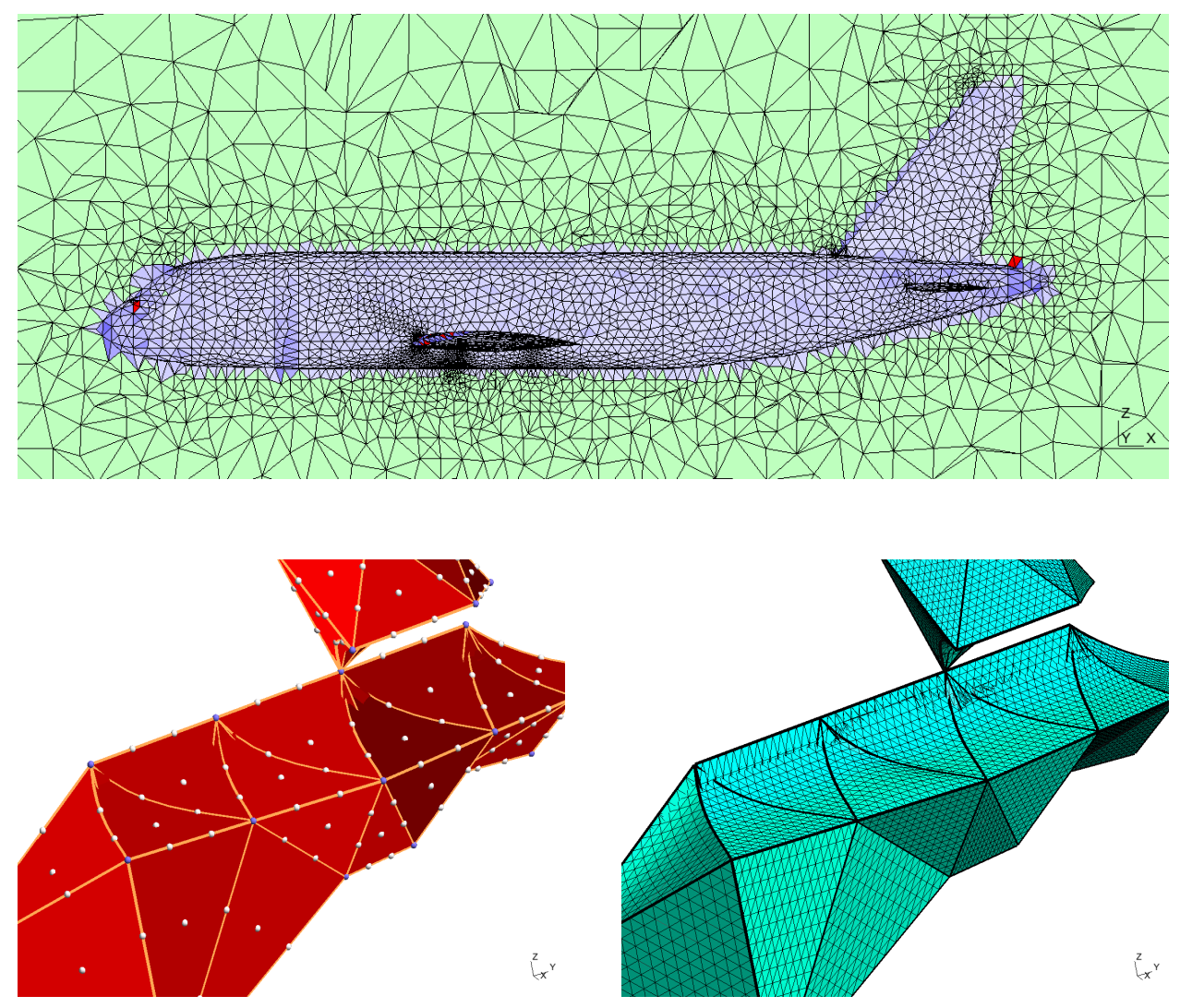

$\delta$

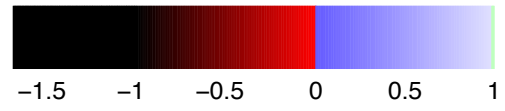

Figure 8: The top figure shows a cut of the mesh of the A319 plane (figure 7). The two bottom figures show same invalid elements. On the left, elements are colored in function of their distortion. Here, we can clearly see that some faces intersect each other. In some cases, the Jacobian determinant is negative only inside the element so as it is not possible to see it visually. 
robust, and a user-defined error tolerance permits to adjust the accuracy vs. computational time ratio.

Perspectives for future work are numerous. We are currently investigating two related areas: first, we are working on a generalization of the bounds presented in this paper to the case of surface meshes embedded in 3D (curved surfaces). Second, we are investigating the use of various optimization strategies to generate meshes with provably good qualities.

\section{Acknowledgement}

This research project was funded in part by the Walloon Region under WIST 3 grant 1017074 (DOMHEX).

Authors gratefully thank E. Bechet from the University of Liège and K. Hillewaert from Cenaero for insightful discussions about Bézier functions and curvilinear mesh generation. Authors also thank V. D. Nguyen for providing the microstructure geometry used in Figure 4.

\section{References}

[1] S. Dey, R. M. O'Bara, M. S. Shephard, Curvilinear mesh generation in 3D, Computer Aided Geom. Design 33 (2001) 199-209.

[2] M. S. Shephard, J. E. Flaherty, K. E. Jansen, X. Li, X. Luo, N. Chevaugeon, J.-F. Remacle, M. W. Beall, R. M. O'Bara, Adaptive mesh generation for curved domains, Applied Numerical Mathematics 52 (2005) 251-271.

[3] S. J. Sherwin, J. Peiró, Mesh generation in curvilinear domains using high-order elements, International Journal for Numerical Methods in Engineering 53 (2002) 207-223.

[4] C. Geuzaine, J.-F. Remacle, Gmsh: a three-dimensional finite element mesh generator with built-in pre- and post-processing facilities, International Journal for Numerical Methods in Engineering 79 (11) (2009) $1309-1331$.

[5] X. Roca, A. Gargallo-Peiró, J. Sarrate, Defining Quality Measures for High-Order Planar Triangles and Curved Mesh Generation, Proceedings of the 20th International Meshing Roundtable (2012) 365-383.

[6] T. Hughes, The Finite Element Method, Dover, 2003. 
[7] I. Babuška, B. Szabó, R. L. Actis, Hierarchic models for laminated composites, International Journal for Numerical Methods in Engineering 33 (1992) 503-535.

[8] G. E. Farin, Curves and surfaces for CAGD: a practicle guide, MorganKaufmann, 2002.

[9] J. M. Lane, R. F. Riesenfeld, A theoretical development for the computer generation and display of piecewise polynomial surfaces, IEEE Transactions on Pattern Analysis and Machine Intelligence 2 (1) (1980) $35-46$.

[10] E. Cohen, L. L. Schumacker, Rates of convergence of control polygons, Computer Aided Geometric Design 2 (1985) 229-235. 